
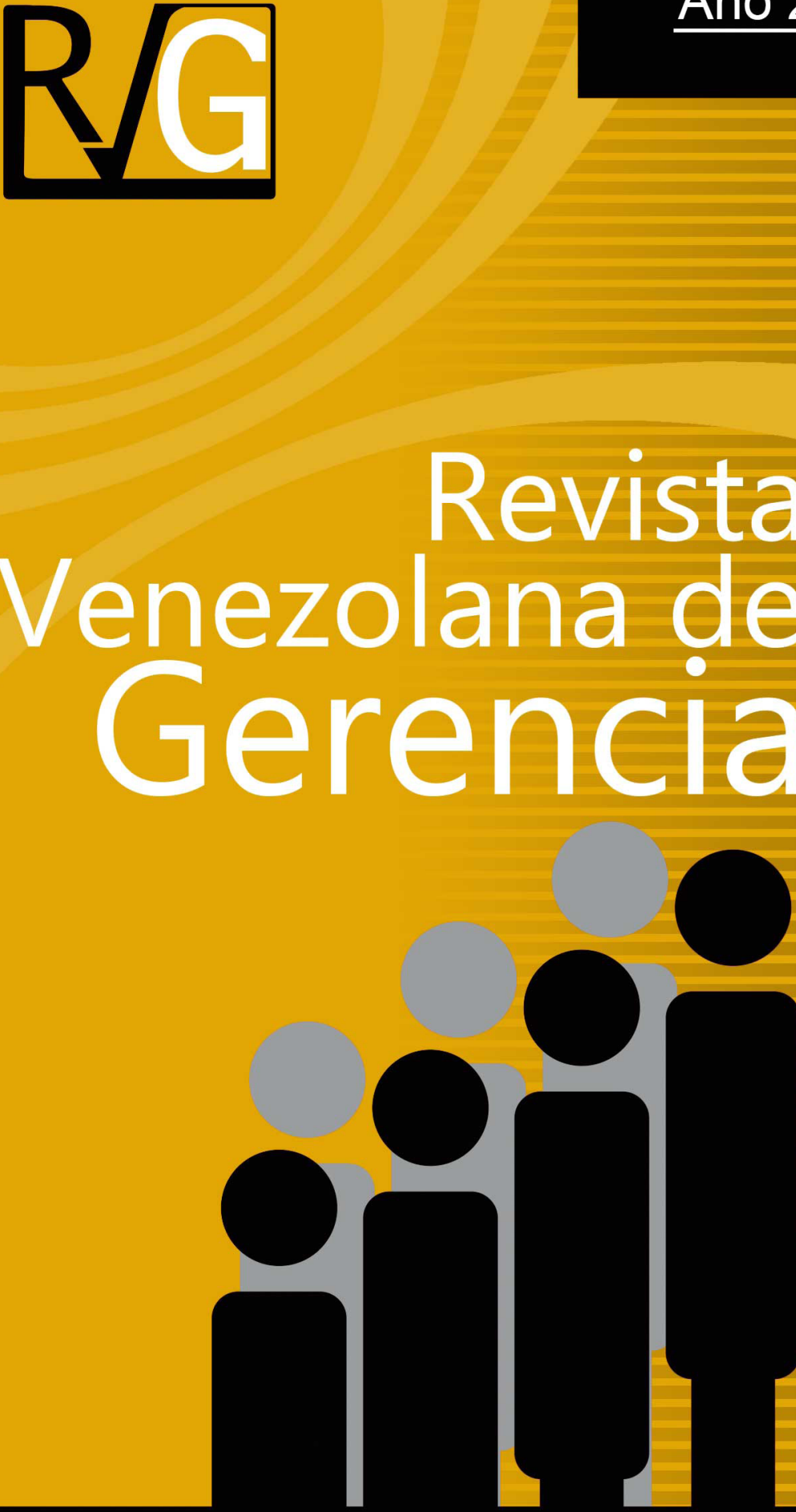


\title{
"Felicidad" asociada al bienestar laboral: categorización de variables
}

\author{
Jiménez Sierra, Devinso' \\ Ortiz Padilla, Myriam² \\ Monsalve Perdomo, Martha ${ }^{3}$ \\ Gómez García, Melchor ${ }^{4}$
}

\section{Resumen}

El artículo es de revisión documental, muestra los modelos de medición de la felicidad desde la perspectiva global y desde los postulados teóricos de investigaciones recientes que abordan la variable de estudio "Felicidad relacionada con el bienestar laboral". Responde la pregunta problema ¿Cuáles son las variables asociadas al concepto de felicidad?. Su enfoque es hermenéutico, la metodología es de tipo cualitativodocumental, en este, se muestra de manera sistemática como identifican las variables dominantes en el tema de la felicidad a partir de su estado del arte y las asociaciones entre categorías madres y subcategorías que se desarrollan como constructos de la variable de estudio. Los resultados arrojan una prevalencia de estudios cualitativos, donde todos los modelos están asociados a 51 variables. Posterior se realizó una depuración pasar a 25 categorías, justificando la creación de un modelo de indexación de felicidad y el bienestar laboral en organizaciones. El estudio permitió mostrar la

\section{Recibido: 20-06-19 Aceptado: 15-10-19}

1 Magister en Educación, Filosofo. Profesor Investigador de la Corporación Unificada Nacional de Educación Superior "CUN" - Santa Marta, Colombia. Email: devinso_jimenez@cun.edu.co ORCID: https://orcid.org/0000-0001-9674-6867

2 Doctora en Educación, Magister en Educación. Psicológica. Directora del Doctorado en Ciencias de la Educación y Profesora Investigadora del Grupo Sinapsis Educativa y Social de la Universidad Simón Bolívar - Barranquilla, Colombia. Email: mortiz@unisimonbolivar.edu.co ORCID: https://orcid.org/0000-0002-9039-0297

3 Magister en Educación, Licenciada en Educación Preescolar. Directora del Centro de Investigación y Líder del Grupo de Investigación Interdisciplinar GRUPINS del Instituto Nacional de Formación Técnica Profesional "Humberto Velásquez García" - Ciénaga, Colombia. Email: marthamonsalve@infotephvg.edu.co ORCID: https://orcid.org/0000-0002-9039-0297

4 Doctor en Filosofía y Educación, Licenciado en Matemáticas. Profesor de la Facultad de Formación de Profesorado y Educación de la Universidad Autónoma de Madrid - Madrid, España. Emil: melchor.gomez@uam.es ORCID: https://orcid.org/0000-0003-3453-218X 
relevancia de la variable de estudio descrita. Se consolida la necesidad de sensibilizar y formar sobre la importancia del bienestar laboral y su búsqueda, loable de la felicidad.

Palabras Clave: felicidad; bienestar laboral; categorización.

\title{
"Happiness" associated with the welfare of the workforce: categorization of variables
}

\begin{abstract}
The article is a documentary review, shows the models for measuring happiness from a global perspective and from the theoretical postulates of recent research that address the study variable "Happiness related to work well-being". Answer the problem question What are the variables associated with the concept of happiness? Its approach is hermeneutical, the methodology is of a qualitative-documentary type, in this, it is shown in a systematic way how the dominant variables in the subject of happiness are identified from their state of the art and the associations between mother categories and subcategories that are develop as constructs of the study variable. The results show a prevalence of qualitative studies, where all the models are associated with 51 variables. Subsequently, a purification was carried out to move to 25 categories, justifying the creation of an indexation model of happiness and labor well-being in organizations. The study allowed showing the relevance of the study variable described. The need to raise awareness and train about the importance of work well-being and its praiseworthy pursuit of happiness is consolidated.
\end{abstract}

Keywords: happiness; work well-being; categorization

\section{Introducción}

Ser feliz es una aspiración universal. Desde los primeros estudios filosóficos, la felicidad ha sido un tema de importancia. Aristóteles definió la felicidad como "el bien supremo del hombre, estipulando que para acceder a esta se necesita cultivar nuestras virtudes y vivir de acuerdo a ellas" (Margot, 2007:41). En el siglo XX el filósofo francés Planells escribió: "Todos los hombres buscan la manera de ser felices. Esto no tiene excepción, es el motivo de todos los actos de todos los hombres, hasta de aquellos que se ahorcan" (Planells, 1988:58). En los tiempos modernos, la creciente cantidad de libros de autoayuda presentes en las librerías y la existencia de cada vez más estudiosos enfocados en desarrollar este tema reafirman la necesidad de entender el concepto de felicidad. Esto con el fin de volverlo más tangible y alcanzable.

La constitución de una empresa es una condición de creación de valor, valor que se genera tanto en forma económica 
como en su forma de valor social, muchas empresas deciden devolver, en forma de acciones sociales, parte de los beneficios obtenidos en sus actividades. En muchos casos, la creación de valor social es un hecho indirecto o secundario, que se supedita a la generación de valor económico (Austin, Stevenson \& WeiSkillern, 2012). Es importante reconocer la diversidad de conceptos en torno a la felicidad, este caso, se trata de justificar el concepto desde la perspectiva empresarial, particularmente desde el bienestar laboral.

Además, entendiendo que los indicadores económicos comoel producto interno bruto (PIB) no son funcionales para interpretar los niveles de bienestar de los ciudadanos, algunos países han tenido la iniciativa de incorporar índices de felicidad. Esto con el fin de medir su eficacia gubernamental y tener una visión más integral que les permita implementar políticas que viabilicen y faciliten el desarrollo sostenible de un buen vivir para sus ciudadanos. (López y Fierro, 2015). La felicidad no es sólo una disciplina especulativa, representa hoy una vertiente de la sicología positiva desde la visión de una empresa con sentido social.

Desde el desarrollo de este artículo se reconoce la perspectiva en la que se define la empresa y su valor ético en sí mismo que supone conducir al ser humano hacia la excelencia, a ser mejor persona y, por tanto, a alcanzar, aun cuando sea inconscientemente, la felicidad como fin último. Lograr esto en las personas es positivo para la organización, debido a que estas como parte fundamental en la sociedad, sus acciones van a afectar y por tanto deben servir las necesidades de las personas y satisfacerlas (Jiménez y Pérez, 2016). El individuo, al sentir alcanzado su fin último, se sentirá mejor y esto, con toda probabilidad, podría traducirse en un conjunto de actitudes y comportamiento laborales del empleado muy positivo desde una perspectiva organizacional. (Ruiz, Ruiz, y Martínez, 2012).

Esta investigación se justifica desde el punto de vista teórico desde los estudios de la sicología positiva y organizacional de Warr (2013) en ella se analizan por separado las características del entorno y los procesos mentales del individuo. Se examina, además, el funcionamiento combinado de los factores externos e internos vinculado a lo laboral, exponiendo cómo la felicidad puede depender tanto de las características laborales como personales. El autor presenta una serie de sugerencias para futuras investigaciones, entre las cuales se destaca la necesidad de distinguir entre las diferentes formas de felicidad e infelicidad, tanto a nivel conceptual como empírico, ya que cada una tiene sus propias causas y consecuencias. También se incluye la teoría de Layard (Tula, 2015) quien propone la felicidad como ciencias del estudio del comportamiento humano y su preocupación por la felicidad individual como factor asociado al desarrollo y bienestar de las personas en las organizaciones.

En este sentido, el presente estudio tiene como objetivo responder la pregunta problema ¿Cuáles son las variables asociadas al concepto de felicidad? El cual se enmarca dentro del macro-proyecto "Índice de felicidad en relación al bienestar laboral en Pymes de la ciudad de Santa Marta". Este es desarrollado por medio de una revisión documental, puesto que se realiza una revisión al estado del arte en función de la variable de estudio "Felicidad en 
relación con el bienestar laboral”.

\section{Hacia un concepto de felicidad laboral}

La práctica social, en el contexto laboral, es una relación de individuos que coexisten en espacios comunes en busca de resultados afines a la organización, por ello es determinante para todas las personas, llevar una relación amena con las mismas de la forma más armoniosa posible. Se sabe que, esto sin duda alguna contribuye a aumentar la felicidad en uno como ser humano. Así pues, para tener éxito en la búsqueda de la felicidad es importante pensar y actuar desde la satisfacción que produce la acción realizada; su bienestar individual (Percy, 2011).

Experimentar sentimientos positivos induce a la gente a tener éxito en el trabajo y a perseverar en los esfuerzos para alcanzar sus metas, entendiendo que las relaciones interpersonales que se tienen en el trabajo tienen el mayor impacto en nuestra felicidad (Dutschke, 2013). Por tanto, las organizaciones de hoy tratan de evitar la insatisfacción laboral puesto que a menor insatisfacción laboral se obtiene una respuesta positiva orientada al trabajo. En una publicación que revolucionó la perspectiva sobre la felicidad Layard citado por (Tula, 2015) y que posteriormente se convirtió en el best seller "La ciencia de la felicidad" el autor reconoce siete grandes factores que influyen en la felicidad; estos son: 1) relaciones familiares, 2) situación financiera, 3) trabajo, 4) comunidad y amigos, 5) libertad personal y 6) valores personales; 7) remuneración por el trabajo, todos hablan de relaciones.

Para el autor, la calidad y la estabilidad de las relaciones importa más que su forma: se necesita de otras personas y de que otros necesiten de nosotros; cada vez más las investigaciones confirman la máxima importancia del amor (Tula, 2015). Layard (2008) funda su argumentación en una investigación longitudinal en 50 países durante (4) años en los que midió los siete factores de la felicidad en el trabajo. Su conclusión es que seis factores, de los siete definidos en su teoría, explican la variación del $80 \%$ de la felicidad. De modo general, puede decirse que las personas que se preocupan por otros son en media más felices de las que se preocupan por sí mismas. En este sentido, lo que importan no es lo que la vida le ofrece, sino lo que uno ofrece a la vida. (Tula, 2015)

La obra de Layard explora la pregunta: ¿Por qué no se es feliz siendo rico? En su opinión el crecimiento económico no aumenta la felicidad porque en la misma medida en que se eleva, también aumenta le criterio con el que se la juzga. Por otra parte, la renta no es sólo un medio de comprar, sino también de compararse con los otros; de aquí la importancia de la renta relativa: la mayoría prefiere ser más pobres si su posición relativa en su entorno mejora. (Tula, 2015)

Otra perspectiva en el trabajo responde a la teoría de Warr (2013) de acuerdo con este autor, un aspecto fundamental para entender la felicidad en el trabajo es la manera como se clasifican las características laborales, éstas se asocian en categorías madres y subcategorías de la siguiente manera:

1) Oportunidad de control; esta categoría madre está relacionada con las sub-categorías; Influencia personal, autonomía, libertad de decisión, participación, libertad en la toma de decisiones. 2) Oportunidad para el uso y la adquisición de habilidades; y sus sub- 
categorías; Un entorno potencial para el uso y desarrollo de competencias y conocimientos. 3) Metas generadas externamente; responde a la manera como los colaboradores actúan en relación con: Demandas externas, desafío, baja carga y sobrecarga de trabajo, identificación con la tarea, conflicto de rol, trabajo emocional, conflicto trabajo-casa. 4) La Variedad; se suscribe a cambios en el contenido de las tareas y los contactos sociales, localización trabajo variada. 5) Claridad del entorno y Resultados predecibles, requisitos claros, claridad de rol, retroalimentación sobre las tareas, baja ambigüedad respecto al futuro.

6) Relación y contacto con los otros y enmarca el contacto social, calidad en las relaciones sociales, dependencia de los demás, trabajo en equipo. 7) Disponibilidad del dinero y los ingresos disponibles, nivel salarial, pago por resultados. 8) Seguridad física y las condiciones de trabajo adecuadas, grado de riesgos, calidad de los equipos de trabajo. 9 y 10) se asocian a la posición socialmente valorada y al apoyo del supervisor con relación a la consideración por parte de los jefes, trato justo por parte del supervisor y preocupación por el bienestar propio. Las dos últimas categorías responden al desarrollo de carrera y la equidad teniendo en cuenta la justicia dentro de la propia organización, equidad en las relaciones de la organización con la sociedad. (Warr, 2013).

Es importante definir que los postulados teóricos previamente presentados están representados en escenarios organizacionales formales y contextos empresariales ajenos a las condiciones de vida laboral que se abordaran en la investigación, pero responden a una línea base desde la cual se puede ajustar y modelar un instrumento que se ajuste a las condiciones propias del problema de investigación.

Por otro lado; el concepto de bienestar laboral es concebido como una de las variables más importantes en la investigación del comportamiento organizacional al ser uno de los elementos socioculturales de la organización, lo que obliga a su estudio para poder gestionar equipos de trabajo y empresas (Calderón, Laca, Pedroza, y Pando, 2015). Hoy existen numerosas formas de concebir el bienestar psicológico y laboral. Su estudio está relacionado con conceptos tales como el de satisfacción laboral, capital psicológico, fluidez, compromiso y elevación; en suma, todo aquello que hace referencia al funcionamiento óptimo del individuo en el trabajo y que es un reflejo de su percepción de calidad de vida que experimenta. (Calderon, 2016).

No es nuevo que las organizaciones sean estudiadas por la psicología debido a su relevancia en el funcionamiento y el bienestar de los individuos. Entre otras variables, se ha investigado la cultura organizacional y sus dimensiones asociadas al sistema de creencias, normas, tradiciones y costumbres que los miembros de una organización han construido y que rigen su comportamiento, encontrándose que este fenómeno influye sobre la productividad y la satisfacción laboral (Calderon, 2016). Desde esta perspectiva se puede encontrar vasos comunicantes entre la felicidad como comportamiento inherente al ser humano y su relación con el contexto laboral (Rivera-Porras, Rozo-Sánchez, Flórez-Garay, 2018).

Otro de los temas de la investigación son los índices de 
felicidad, este concepto no es nuevo, existe desde diferentes vertientes $y$ puntos de convergencias. (Prieto-Ursúa, 2006; Lupano y Castro, 2010). Uno de los primeros países en desarrollar un modelo basado en índices de felicidad fue Bután. Esta nación en 1929 registró en su código legal que, si un gobierno no crea felicidad para su gente, el propósito de este no existe. (López y Fierro, 2015) Por su parte, en 1972 el rey IV de Bután, su majestad Jigme Singye Wangchuck, ideó por primera vez un Índice de Felicidad Nacional Bruta (IFNB) para medir el bienestar de sus ciudadanos y destacó que este es más importante que el índice de producción y consumo de bienes y productos PIB. En dicha proposición el gobierno de Bután propuso su modelo basado en nueve variables: 1) bienestar, 2) estándares de vida, 3) salud, 4) cultura, 5) educación, 6) vitalidad comunitaria, 7) buen gobierno, 8) uso del tiempo balanceado y 9) integración ecológica. (Dutschke, 2013).

\section{Metodología para la identificación de variables asociadas al concepto de felicidad}

Para lograr el objetivo de este análisis, la investigación se orientó en una metodología Cualitativa que, según Hernández, Fernández y Baptista (2014), se fundamenta en una perspectiva interpretativa centrada en el entendimiento del significado de las acciones de seres vivos, sobre todo de los humanos y sus instituciones. Se enfocó en una revisión documental, puesto que se realiza una revisión al estado del arte en función de la variable de estudio "Felicidad en relación con el bienestar laboral". Es necesario enunciar que la recolección de información para esta investigación se hizo a través de tres instrumentos con el objetivo de identificar las categorías relevantes en los modelos de medición de la felicidad y la literatura relevante en consonancia con la variable de estudio. En un primer momento se hizo uso de una matriz de análisis documental construida para este estudio. En segundo momento, el análisis cualitativo de la información sistematizada se hizo por medio del software Atlas_ti V7 y finalmente en un tercer momento una matriz de expertos bajo el Método Delphi. Con el primer instrumento se filtraron los artículos de la base de datos de Redalyc.org con mayor coeficiente H5, lo que arrojó 14 artículos que fueron objeto de análisis. Con el apoyo de un software de análisis cualitativo se asociaron las categorías objeto de estudio en categorías madres y subcategorías. En el tercer momento; bajo el método Delphi y el concurso de siete jueces expertos se determinó cuáles de las variables se ajustaban al constructo teórico de la variable de estudio.

\section{Felicidad relacionada con el bienestar laboral}

El gráfico 1 muestra la incidencia de las publicaciones asociadas a las categorías "trabajo", "felicidad" e "índice", tomando como referente la base de datos de la Red Académica de Latinoamérica y el Caribe (Redalyc). Las categorías muestran un uso ascendente y constante hasta el año 2013, dos leves descensos en el año 2014 y 2016 y un repunte para el año 2017 siendo el año con mayores publicaciones asociadas a las categorías en revisión. Se tomaron artículos científicos desde el año 2008 hasta el año 2017. Los años 2018 y 
2019 no fueron tenidos en cuenta en la revisión, ya que entre estos fue realizada la recolección de los datos, su análisis y determinación de resultados. Esto muestra que el tema de la felicidad asociada al trabajo es un tema de estudio en el ámbito de la administración de empresas.

\section{Gráfico 1}

\section{Incidencia de publicaciones en artículos científicos base de datos Redalyc}

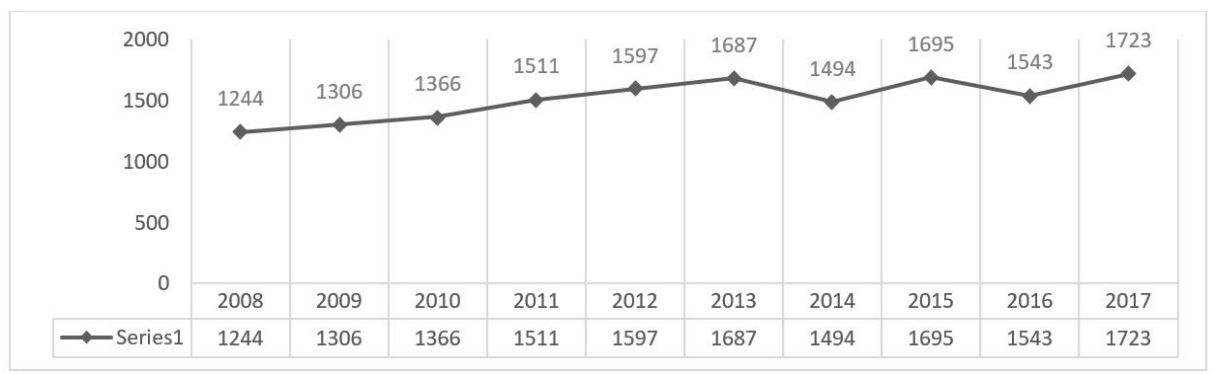

Fuente: Elaboración propia

Después de identificar la incidencia del tema de felicidad en el trabajo se procedió a reconocer los modelos generales que han marcado la medición de felicidad desde una perspectiva global, se definieron sólo los macro-modelos que tenían impacto mundial. El cuadro 1, muestra que la primera vez que se diseñó un modelo de medición macro-estructural de la felicidad era el año 1972, promovido por el entonces gobernante de Bután y que recibió el nombre de Modelo Batún, un país del sur de asía, que incorporó las variables asociadas a la felicidad y contrastarlo con sus índices de PIB. En el año 2006 surgió el Happy Planet Index de la fundación NEF que incorporó las categorías asociadas a huella ecológica. En el año 2011 la OCDE formula la Better Life Index como un mecanismo de medir el bienestar general de sus países miembros, el DANE en Colombia incorporó la encuesta nacional de bienestar de vida desde la misma directriz. En el año 2013, Porter y Stern formulan el modelo Social Progres Index. 


\begin{tabular}{ccc} 
& \multicolumn{2}{c}{$\begin{array}{c}\text { Cuadro 1 } \\
\text { Modelos de indexación de la felicidad }\end{array}$} \\
\hline \multicolumn{1}{c}{ Modelos } & \multicolumn{1}{c}{ Autor / Año } & \multicolumn{1}{c}{ Variables } \\
\hline $\begin{array}{l}\text { Modelo de medición de } \\
\text { felicidad de Bután }\end{array}$ & \multicolumn{1}{c}{$\begin{array}{c}\text { Bienestar psicológico /Uso del tiempo/ } \\
\text { Jigme Singye Wangchuck (1972) }\end{array}$} & $\begin{array}{l}\text { Vitalidad de la comunidad/Cultura/Salud/ } \\
\text { Educación/Diversidad ambiental/Nivel de } \\
\text { vida/Relación con el gobierno }\end{array}$ \\
\hline Happy Planet Index & $\begin{array}{l}\text { New Economics Foundation } \\
\text { (NEF) (2006) }\end{array}$ & $\begin{array}{l}\text { Grado de satisfacción del bienestar/ } \\
\text { expectativa de vida / huella ecológica }\end{array}$ \\
\hline Better Life Índex & OCDE - Joseph Stiglitz (2011) & $\begin{array}{l}\text { Vivienda/Ingresos/Empleo/Comunidad/ } \\
\text { Educación/Medio ambiente/Compromiso } \\
\text { cívico/Salud/Satisfacción/ } \\
\text { Equilibrio entre vida y trabajo }\end{array}$ \\
\hline Social Progress Index & $\begin{array}{l}\text { Michael Porter-Scott Stern } \\
\text { (2013) }\end{array}$ & $\begin{array}{l}\text { Necesidades humanas básicas/bienestar } \\
\text { fundamental / oportunidades de progreso. }\end{array}$ \\
\hline
\end{tabular}

Fuente: Elaboración propia

Al relacionar las variables de estudio de los distintos modelos de indexación de la felicidad, se realizó un análisis para determinar la relación entre ellos. Apoyado en una escala cromática desde un software cualitativo se identificaron las variables con mayor asociación siendo la escala del rojo la más asociada y la escala del azul el menos asociado. Esto mostró que las categorías "Grado de satisfacción" "nivel de vida" y "bienestar psicológico" son los de mayor asociación. Le siguen "huella de carbón", "medio ambiente" y "relaciones con el gobierno" "oportunidades de progreso", "empleo" y "seguridad". En un tercer bloque "comunidad", "salud", "expectativa de vida" "satisfacción" entre otras. En un último las menos asociadas son "vivienda", "vitalidad de la comunidad" y "cultura", En el diagrama 1 se muestra la red semántica como herramienta hermenéutica que permite realizar asociaciones entre categorías. Se presentan las interacciones existentes entre las variables descritas en los modelos de medición de la felicidad descritos en el cuadro 1.

En el cuadro 2, se describieron las investigaciones de los últimos años con mayor índice $\mathrm{H} 5$, que proponen un modelo explicativo asociado a felicidad en el trabajo. Para ello se utilizó una matriz de análisis documental para indagar tres aspectos; autores/Variables asociadas/Enfoque metodológico. Sólo se tomaron investigaciones científicas de las cuales se filtraron 14 investigaciones. De estas, cinco tenían enfoques mixtos (cualitativo-cuantitativo) y nueve de enfoque cualitativo, lo que muestra que la mayoría de las investigaciones asociadas a la felicidad laboral son de enfoque cualitativo. El análisis arrojó que todos estos modelos explicativos están asociados a 51 variables. (Ver Cuadro 2). 


\section{Diagrama 1 \\ Red semántica: categorización de variables}

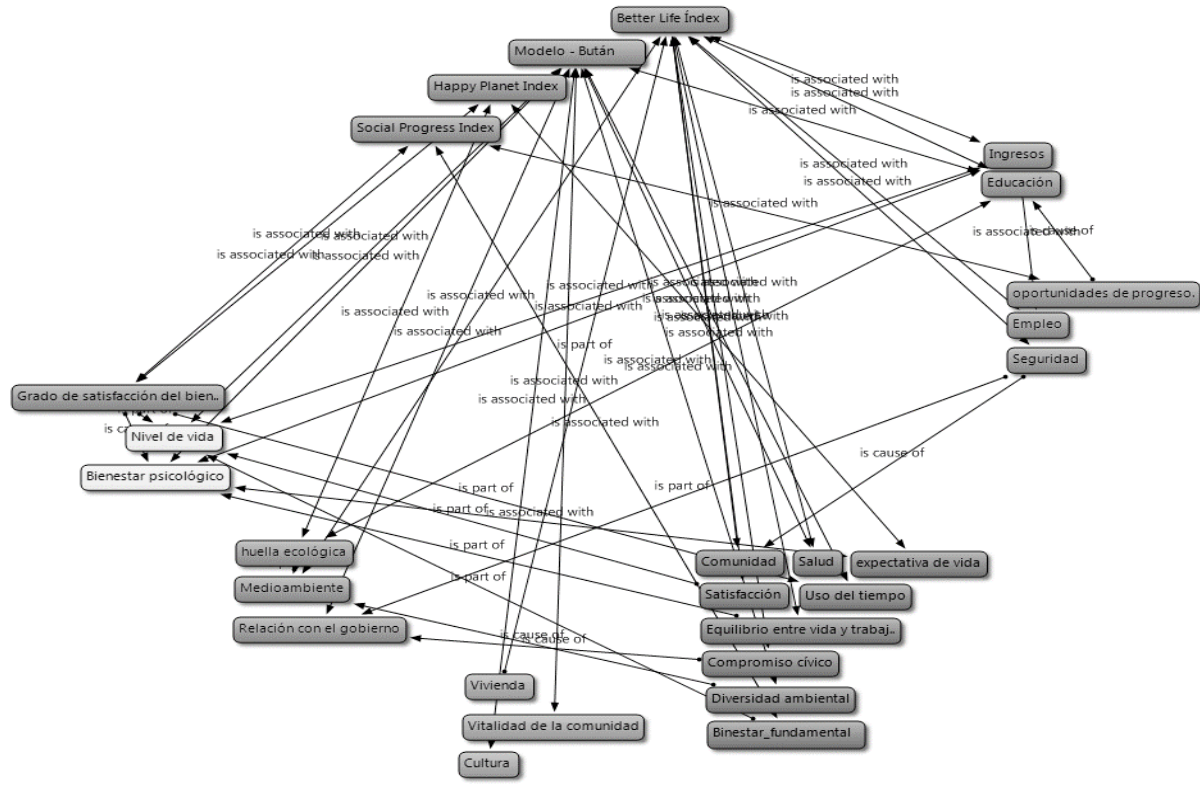

Fuente: elaboración propia

\section{Cuadro 2}

Modelos explicativos asociados a la felicidad en el trabajo

\begin{tabular}{|c|c|c|c|c|}
\hline $\mathrm{N}^{\circ}$ & Autor/es & Investigación & Variables & Enfoque \\
\hline 1 & Urarte (2009) & La felicidad en el Trabajo & $\begin{array}{l}\text { Equilibrio trabajo-vida /Calidad } \\
\text { de vida laboral / Satisfacción } \\
\text { laboral /Actitudes hacia el } \\
\text { trabajo/ Calidad de vida / } \\
\text { Felicidad }\end{array}$ & Cualitativa \\
\hline 2 & Gamero (2013) & $\begin{array}{l}\text { La Satisfacción Laboral } \\
\text { como Dimensión de la } \\
\text { Felicidad }\end{array}$ & $\begin{array}{l}\text { modelo de interacción } \\
\text { / satisfacción laboral / } \\
\text { felicidad }\end{array}$ & Cuali-cuantitativa \\
\hline 3 & Warr (2013) & $\begin{array}{l}\text { Fuentes de felicidad e in- } \\
\text { felicidad en el trabajo: una } \\
\text { perspectiva combinada }\end{array}$ & $\begin{array}{l}\text { Procesos mentales caracte- } \\
\text { rísticas del entorno / procesos } \\
\text { cognitivos / procesos afectivos }\end{array}$ & Cuali-cuantitativa \\
\hline 4 & $\begin{array}{l}\text { Rodríguez-Muñoz } \\
\text { y Sanz (2013) }\end{array}$ & $\begin{array}{l}\text { Felicidad y bienestar en } \\
\text { el trabajo: introducción al } \\
\text { número especial }\end{array}$ & $\begin{array}{l}\text { Bienestar en el trabajo/ Satis- } \\
\text { facción / felicidad }\end{array}$ & Cualitativa \\
\hline
\end{tabular}




\section{Cont... Cuadro 2}

\begin{tabular}{|c|c|c|c|c|}
\hline 5 & $\begin{array}{l}\text { Nader, Peña, y } \\
\text { Sánchez (2014) }\end{array}$ & $\begin{array}{l}\text { Predicción de la satisfac- } \\
\text { ción y el bienestar en el } \\
\text { trabajo: hacia un modelo de } \\
\text { organización saludable en } \\
\text { Colombia }\end{array}$ & Clima laboral / satisfacción & Cualitativa \\
\hline 6 & $\begin{array}{l}\text { Jiménez, Martín y } \\
\text { Montero (2014) }\end{array}$ & $\begin{array}{l}\text { Felicidad, desempleo y } \\
\text { crisis económica en Anda- } \\
\text { lucía. Algunas evidencias }\end{array}$ & $\begin{array}{l}\text { Desempleo / condiciones de } \\
\text { vida / mercado de trabajo }\end{array}$ & Cuali-cuantitativa \\
\hline 7 & $\begin{array}{l}\text { Veloso, Cuadra, } \\
\text { Gil, Quiroz y Meza } \\
(2015)\end{array}$ & $\begin{array}{l}\text { Capacitación en trabajado- } \\
\text { res: impacto de un progra- } \\
\text { ma, basado en psicología } \\
\text { positiva y habilidades } \\
\text { sociales, en satisfacción } \\
\text { vital, satisfacción laboral y } \\
\text { clima organizacional }\end{array}$ & $\begin{array}{l}\text { Habilidades sociales / psico- } \\
\text { logía positiva / trabajadores / } \\
\text { satisfacción }\end{array}$ & Cuali-cuantitativa \\
\hline 8 & $\begin{array}{l}\text { Madero y Barboza } \\
(2015)\end{array}$ & $\begin{array}{l}\text { Interrelación de la cultura, } \\
\text { flexibilidad laboral, alinea- } \\
\text { ción estratégica, innovación } \\
\text { y rendimiento empresarial }\end{array}$ & $\begin{array}{l}\text { Flexibilidad organizacional /Ali- } \\
\text { neación estratégica / Cultura } \\
\text { organizacional /Innovación / } \\
\text { Desempeño de la empresa }\end{array}$ & Cualitativa \\
\hline 9 & $\begin{array}{l}\text { De Sousa y Porto } \\
\text { Barreiros (2015) }\end{array}$ & $\begin{array}{l}\text { La Felicidad Laboral: El } \\
\text { Impacto de los Valores Or- } \\
\text { ganizacionales y del Ajuste } \\
\text { Persona-Organización }\end{array}$ & $\begin{array}{l}\text { Conducta organizacional / } \\
\text { felicidad / teoría de los valores }\end{array}$ & Cualitativa \\
\hline 10 & $\begin{array}{l}\text { Cabanas \& } \\
\text { Sánchez (2016) }\end{array}$ & $\begin{array}{l}\text { Invirtiendo la pirámide de } \\
\text { las necesidades: la psico- } \\
\text { logía positiva y la nueva } \\
\text { lógica del éxito laboral }\end{array}$ & $\begin{array}{l}\text { Psicología positiva/psicología } \\
\text { del trabajo/felicidad/pirámide } \\
\text { de las necesidades/ }\end{array}$ & Cualitativa \\
\hline 11 & Fratocchi (2016) & Felicidad y trabajo. & $\begin{array}{l}\text { Mercado laboral / felicidad } \\
\text { laboral }\end{array}$ & Cualitativa \\
\hline 12 & $\begin{array}{l}\text { Domínguez e } \\
\text { lbarra (2017) }\end{array}$ & $\begin{array}{l}\text { La psicología positiva: Un } \\
\text { nuevo enfoque para el } \\
\text { estudio de la felicidad }\end{array}$ & $\begin{array}{l}\text { Potencial humano / bienestar / } \\
\text { psicología positiva }\end{array}$ & Cualitativa \\
\hline 13 & $\begin{array}{l}\text { Satuf y Pires } \\
(2017)\end{array}$ & $\begin{array}{l}\text { Satisfacción laboral y feli- } \\
\text { cidad: Un estudio compa- } \\
\text { rativo entre portugueses y } \\
\text { brasileros. }\end{array}$ & $\begin{array}{l}\text { Gestión de personas / satis- } \\
\text { facción laboral / emociones } \\
\text { positivas }\end{array}$ & Cualitativa \\
\hline 14 & $\begin{array}{l}\text { Pujol-Cols y Da- } \\
\text { bos (2018) }\end{array}$ & $\begin{array}{l}\text { Satisfacción laboral: una } \\
\text { revisión de la literatura } \\
\text { acerca de sus principales } \\
\text { determinantes }\end{array}$ & $\begin{array}{l}\text { satisfacción laboral / diseño } \\
\text { del trabajo }\end{array}$ & Cuali-cuantitativa \\
\hline
\end{tabular}

Fuente: elaboración Propia

En el diagrama 2 se asociaron y eliminaron las categorías repetidas para depurar las variables de estudio que contengan, de modo explicativo, la categoría madre de esta investigación; "felicidad asociada al bienestar laboral" para ello se utilizó los juicios de 3 jueces expertos quienes agruparon 
en asocio con categorías madres, las variables de los modelos explicativos del análisis documental. Se catalogaron 13 categorías madre, de las cuales 10 tienes sud-categorías asociadas, a saber; "satisfacción", "actitudes para el trabajo", "felicidad", "Psicología positiva", "equilibrio trabajo-vida", "clima laboral", "calidad de vida", "emociones positivas", "procesos cognitivos" y "mercado laboral". Y tres categorías madre sin subcategorías asociadas, a saber; "innovación", "trabajadores" y "desempleo" (diagrama 2).

\section{Diagrama 2 \\ Red semántica: categorización de variables de los modelos explicativos del estado del arte (2018)}

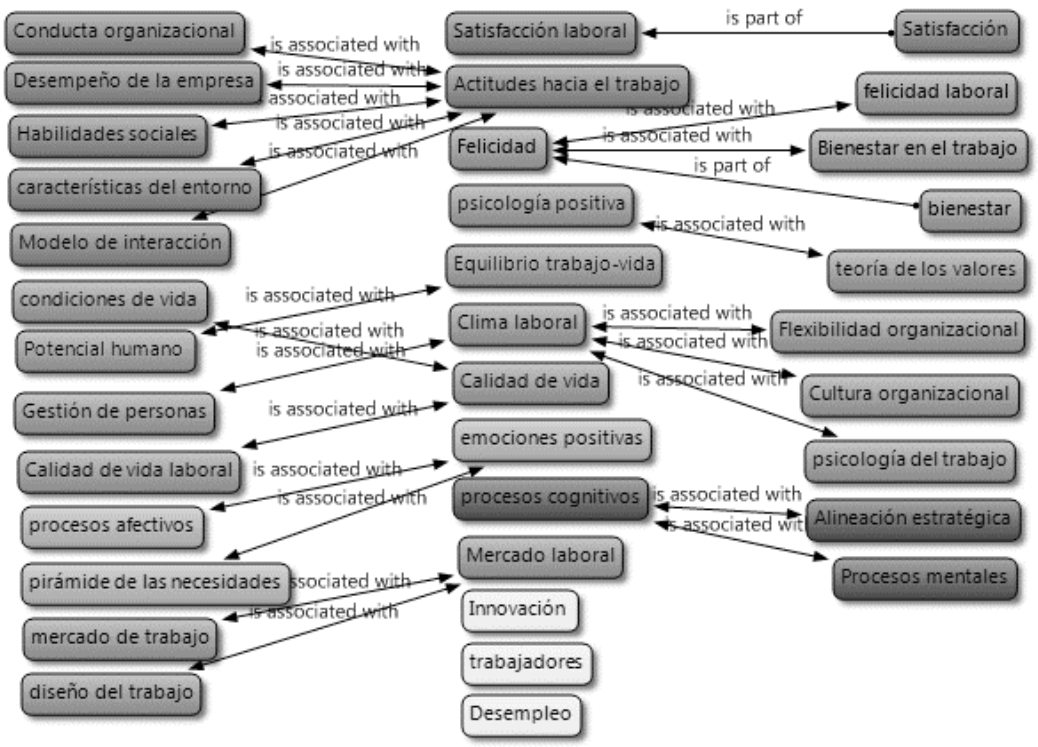

Fuente: elaboración propia

La tabla 1, muestra las valoraciones que hicieron siete jueces expertos por medio del método Delphi, para determinar el grado en que las categorías madres estaban contenidas en la variable de estudio, a saber; felicidad en el bienestar laboral, para ello se diligenció una matriz en la que se valoraba su relación en una escala de uno (1) a cinco (5). Así se constató, que, a juicios de los jueces expertos, que sólo las categorías madres "clima laboral", "felicidad", "satisfacción laboral", "calidad de vida", "emociones positivas", actividades hacia el trabajo" y "equilibrio trabajo-vida" están significativamente asociadas a la variable de estudio. 


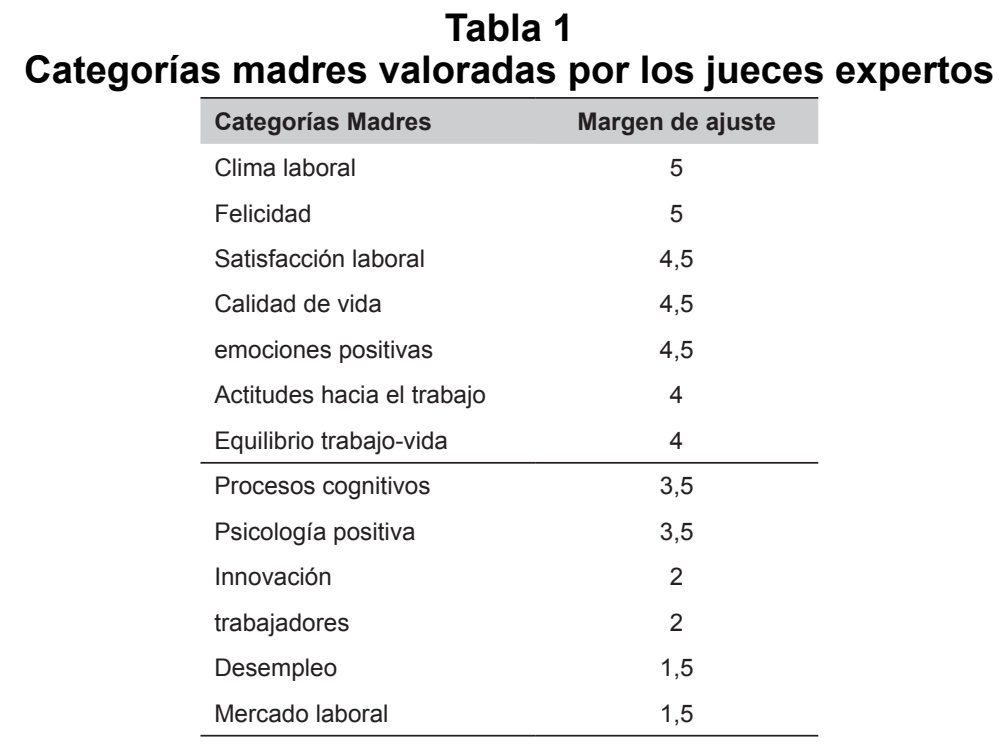

Fuente: Elaboración propia

\section{Diagrama 3 \\ Red semántica: jerarquía de variables de los modelos explicativos del estado del arte}

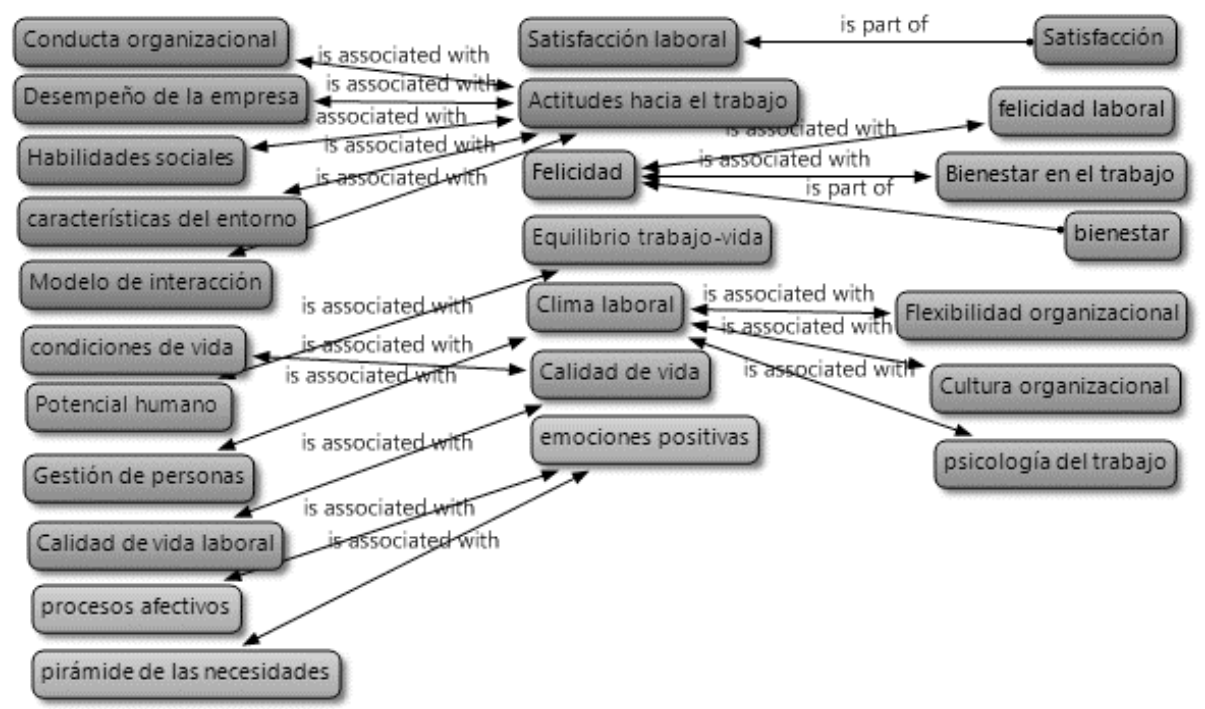

Fuente: elaboración propia 
El diagrama 3, muestra la distribución final de las categorías madres y sus subcategorías asociadas, donde sobresalen "Actitudes hacía el Trabajo" y "Clima Laboral" como las categorías con mayor asociación con otras categorías. Todas las categorías presentadas cuentan con asociación con otras, a excepción de "Satisfacción" y "Bienestar", quienes hacen parte de "Satisfacción Laboral" y "Felicidad" respectivamente, así como "Equilibrio Vida-Trabajo" representada como categoría sin asociación o parte de otra. Esta depuración nos permitió pasar de 51 categorías a 25. Con esta construcción se justifica la creación de un modelo de indexación de la felicidad y el bienestar laboral el cual puede ser usado en diversas organizaciones.

\section{Conclusiones}

Los modelos de indexación de la felicidad han permitido reconocer el concepto "Felicidad" como un elemento que se puede incorporar a la discusión en distintas disciplinas del saber cómo la psicología organizacional y la administración de empresas. En este sentido, dentro de los modelos de indexación de la felicidad que se analizaron en primer momento del estudio, el modelo "Better Life Index" es el que mayor relación guarda con las categorías generadas después del proceso metodológico de la investigación.

Por otro lado, se pudo identificar que el concepto "Felicidad" asociada al bienestar laboral comprende una relación cada vez más estudiada en el ámbito de la administración de empresas, como se puede analizar en la Gráfica No 1 y evidenciado en los datos del Cuadro 2, constituyéndose un acervo probatorio que justifican la investigación en esta problemática creciente en el mundo de las organizaciones. Así mismo, muestra la relevancia teórica de la variable de estudio descrita en este artículo.

En el estudio, se pudieron determinar así, 25 categorías entorno a la variable de "Felicidad" asociada al bienestar laboral, como son: "Conducta Organizacional", "Desempeño de la Empresa", "Habilidades Sociales", "Características del Entorno", "Modelo de Interacción", "Condiciones de Vida", "Características del Entorno", "Modelo de Interacción", "Condiciones de Vida", "Potencial Humano", "Gestión de las Personas", "Calidad de Vida Laboral", "Procesos Afectivos", "Pirámide de las Necesidades", "Mercado de Trabajo", "Diseño del Trabajo", "Satisfacción Laboral", "Actitudes hacia el Trabajo", "Felicidad", "Psicología Positiva", "Equilibrio Trabajo-Vida", "Clima Laboral", "Calidad de Vida", "Emociones Positivas", "Procesos Cognitivos", "Mercado Laboral", "Satisfacción", "Felicidad Laboral", "Bienestar en el Trabajo", "Bienestar", "Teoría de los Valores", "Flexibilidad Organizacional", "Cultura Organizacional”, "Psicología del Trabajo", "Alineación Estratégica" y "Procesos Mentales".

Es así, que se puede afirmar que existen algunas categorías relevantes en torno a la variable "Felicidad" que se ajustan de manera consistente con nuestra realidad organizacional y las dinámicas de competitividad actuales, lo que permite trascender la discusión en torno a la "Felicidad" más allá de los enfoques meramente filosóficos, consolidando la necesidad de sensibilizar y formar sobre la importancia del bienestar laboral y su búsqueda, loable de la felicidad. 


\section{Referencias bibliográficas}

Austin, J., Stevenson, H. y Wei-Skillern, J. (2012). Social and commercial entrepreneurship: same, different, or both?. Revista de Administração, 47(3).

Cabanas, E. y Sánchez-González, J. (2016). Inverting the pyramid of needs: Positive psychology's new order for labor success. Psicothema, 28(2), 107-113.

Calderón, J., Laca, F., Pedroza, F. y Pando, M. (2015). Relación de la socialización organizacional y el compromiso organizacional en trabajadores mexicanos. Psicogente, 18(34), 267-277.

De Sousa, J. y Porto, J. (2015). Happiness at Work: Organizational Values and Person-Organization Fit Impact. Paidéia (Ribeirão Preto), 25(61), pp 211-220

Domínguez, R. y Ibarra, E. (2017). La psicología positiva: Un nuevo enfoque para el estudio de la felicidad. Razón y Palabra, 21(96), 660-679.

Dutschke, G. (2013). Factores condicionantes de felicidad organizacional. Estudio exploratorio de la realidad. Revista de Estudios Empresariales. Segunda época, (1), 21-43.

Fratocchi, V. (2016). Felicidad y trabajo. IEEM Revista de Negocios, 19(3), 44-47.

Gamero, H. (2013). La Satisfacción Laboral como Dimensión de la Felicidad. Ciencia \& Trabajo, 15(47), 94-102.

Hernández, R., Fernández, C. y Baptista, M. (2014). Metodología de la Investigación. México, Mac Graw Hill.

Jiménez, J., Martín, J. y Montero, R.
(2014). Felicidad, desempleo y crisis económica en Andalucía. Algunas evidencias. Revista de Estudios Regionales, (99), 183-207.

Jiménez, D. y Pérez, L. (2016b). La representación Simbólica de la RSE: el Caso Santa Marta. Investigación e Innovación en Ingenierías, 4(2), 2439.

López, J. y Fierro, I. (2015). Determinantes de la felicidad en los administradores: una investigación realizada en las farmacias del Grupo Difare en Ecuador, Universidad \& Empresa, 17(29), 181-211.

Lupano, M. y Castro, A. (2010). Psicología positiva: análisis desde su surgimiento. Ciencias psicológicas, 4(9), 43-56.

Madero, S. y Barboza, G. (2015). Interrelación de la cultura, flexibilidad laboral, alineación estratégica, innovación y rendimiento empresarial. Contaduría y Administración, 60(4), 735-756.

Margot, J. (2007). La Felicidad. Revista Praxis Filosófica, (25), 55-79.

Nader, M., Peña, S. y Sánchez, E. (2014). Predicción de la satisfacción y el bienestar en el trabajo: hacia un modelo de organización saludable en Colombia. Estudios Gerenciales, 30(130), 31-39.

Percy, A. (2011). El coaching de Oscar Wilde: 99 píldoras de sabiduría para la felicidad de aquí. Debolsillo, Madrid.

Planells, J. (1988). Representación y expresión en la filosofía de Schopenhauer. Tesis doctoral, Universitat de València, España, pp 125.

Prieto-Ursúa, M. (2006). Psicología Positiva: una moda polémica. Clínica y Salud, 17(3), 319-338. 
Porter, M.E, Stern, S. y Artavia Loría, R. (2013). Social Progress Index 2013. Washington: Social Progress Imperative.

Pujol-Cols, L. y Dabos, G. (2018). Satisfacción laboral: una revisión de la literatura acerca de sus principales determinantes. Estudios Gerenciales, 34(146), 3-18

Rivera-Porras, D., Rozo-Sánchez, A. y Flórez-Garay, A. (Ed.) (2018). Bienestar psicológico en las organizaciones: Un análisis desde los sectores económicos $y$ productivos. Ediciones Universidad Simón Bolívar, Colombia.

Rodríguez-Muñoz, A. y Sanz-Vergel, A. (2013). Happiness and wellbeing at work: a special issue introduction. Revista de Psicología del Trabajo y de las Organizaciones, 29(3), 95-97.

Ruiz, P., Ruiz, C. y Martínez, R. (2012). Cultura Organizacional Ética y Generación De Valor Sostenible. Investigaciones Europeas de Dirección y Economía de la Empresa, 18(1), 17-31.
Satuf, C. y Reis, I. (2017). Satisfação laboral e felicidade: um estudo comparativo entre portugueses e brasileiros. Revista Psicología Organizações e Trabalho, 17(4), 201-209.

Tula, F. (2015). Felicidad: lecciones de una nueva ciencia LAYARD, Richard. Felicidade: lições de uma nova ciencia, Best Seller, 2008. Sociologías, 17( 40), 416-425

Urarte, Marcos (2009). La felicidad en el trabajo. Revista De Antiguos Alumnos Del IEEM, 12(2), 13-15.

Veloso-Besio, C., Cuadra-Peralta, A., GilRodríguez, F., Quiroz-Cornejo, Á. y Meza-Castro, S. (2015). Capacitación en trabajadores: impacto de un programa, basado en psicología positiva y habilidades sociales, en satisfacción vital, satisfacción laboral y clima organizacional. Interciencia, 40(11), 736-743.

Warr, P.(2013). Fuentes de felicidad e infelicidad en el trabajo: una perspectiva combinada. Revista de Psicología del Trabajo y de las Organizaciones, 29(3), 99-106.

- Esta obra está bajo una licencia de Creative Commons Reconocimiento-NoComercialCompartirlgual 3.0 Unported. http://creativecommons.org/licenses/by-nc-sa/3.0/deed.es_ES 